\title{
Gross Hematuria
}

National Cancer Institute

\section{Source}

National Cancer Institute. Gross Hematuria. NCI Thesaurus. Code C114702.

The visible presence of blood in the urine. 